\title{
The novel mutation p.Asp251Asn in the $\beta$-subunit of succinate-CoA ligase causes encephalomyopathy and elevated succinylcarnitine
}

\author{
Elham Jaberi ${ }^{1}$, Fereshteh Chitsazian ${ }^{2}$, Gholam Ali Shahidi ${ }^{3}$, Mohammad Rohani ${ }^{3}$, Farzad Sina ${ }^{3}$, Iman Safari ${ }^{1}$, \\ Maryam Malakouti Nejad ${ }^{4}$, Masoud Houshmand ${ }^{5}$, Brandy Klotzle ${ }^{6}$ and Elahe Elahi ${ }^{1,4,7}$
}

\begin{abstract}
SUCLA2 is one of several nuclear-encoded genes that can cause encephalomyopathy accompanied by mitochondrial DNA depletion. The disorder usually manifests in early childhood and leads to early death. The gene encodes one of the subunits of succinyl-CoA synthase, the enzyme that catalyzes the reversible conversion of substrates succinyl-CoA and ADP to products succinate and ATP in the tricarboxylic acid pathway. Thirty-two individuals harboring mutations in SUCLA2 have so far been reported, and five different mutations were observed among these individuals. Here we report identification of a novel mutation in SUCLA2 in two cousins affected with encephalomyopathy. The novel mutation causes p.Asp251Asn; the affected amino acid is likely positioned within the ATP-grasp domain of the encoded protein. As previously reported in other patients, we did not observe elevation of methylmalonic acid, the biochemical hallmark of patients with mutations in SUCLA2. We instead found elevated levels of succinylcarnitine.
\end{abstract}

Journal of Human Genetics (2013) 58, 526-530; doi:10.1038/jhg.2013.45; published online 13 June 2013

Keywords: encephalomyopathy; methylmalonic acid; mitochondrial DNA depletion; p.Asp251Asn; succinylcarnitine; SUCLA2

\section{INTRODUCTION}

Mitochondrial diseases constitute a heterogeneous group of disorders with an estimated prevalence of 11.5:100 000. ${ }^{1}$ They can be caused by mutations in mitochondrial DNA (mtDNA) or in nuclear genes. ${ }^{2-6}$ While some of the mutations in the nuclear genes affect the functions of their encoded mitochondrial proteins, others reduce the stability or quantity of mtDNA. ${ }^{7}$ The clinical manifestations of the disorders caused by mitochondrial genomic disruptions are usually myopathic, encephalomyopathic or hepatocerebral; ophthalmic presentations are less frequent. $^{8}$ Onset of symptoms in individuals with mtDNA depletion occurs usually very early in life, and most patients die in early childhood.

The mechanisms that maintain mitochondrial genome stability and regulate mtDNA copy number are not well understood..$^{9}$ Nevertheless, most genes known to affect mtDNA stability and numbers in diseased individuals have biochemical functions that seem relevant to these features of mtDNA. SUCLG1 and SUCLA2 are among the exceptions. These genes encode subunits of succinate-CoA ligase. Succinate-CoA ligase is a heterodimer that occurs in two forms, G-SUCL and A-SUCL. The two forms share a common $\alpha$-subunit encoded by SUCLG1 and have a variant $\beta$-subunit encoded by either SUCLG2 or
SUCLA2. ${ }^{10}$ G-SUCL catalyzes the conversion of succinyl-CoA and GDP to succinate and GTP, and A-SUCL catalyzes the conversion of succinyl-CoA and ADP to succinate and ATP. Both reactions are reversible. A-SUCL is a component of the TCA cycle and G-SUCL may also participate in the pathway; A-SUCL may have additional functions. ${ }^{11,12}$ Both enzymes are localized in the mitochondrial matrix. Their tissue distributions differ, A-SUCL being expressed mostly in the brain and skeletal muscle, which are mainly catabolic tissues, and G-SUCL expressed predominantly in the liver and other anabolic tissues. ${ }^{13,14}$ It has been suggested that G-SUCL and A-SUCL mutations affect mtDNA numbers by affecting the dNTP pool, just as several other mtDNA depletion causing genes appear to do. This suggestion is based on observations of interactions between both forms of the enzyme with nucleoside diphosphate kinase (NDPK), an enzyme involved in the dNTP salvage pathway during mtDNA replication. ${ }^{8,13,15-17}$

In addition to mtDNA depletion in muscle tissue, the common clinical, brain MRI imaging and biochemistry features of patients with mutations in SUCLA2 include, respectively, hypotonia, dystonia, encephalopathy, feeding problems; hyper-signaling in the caudate and putamen nuclei; and lactic acidemia. With respect to biochemical

${ }^{1}$ School of Biology, College of Science, University of Tehran, Tehran, Iran; ${ }^{2}$ Department of Biological Sciences and the Bioformatics Center, Institute of Biochemistry and Biophysics, University of Tehran, Tehran, Iran; ${ }^{3}$ Department of Neurology, Tehran University of Medical Sciences, Tehran, Iran; ${ }^{4}$ Department of Biotechnology, College of Science,

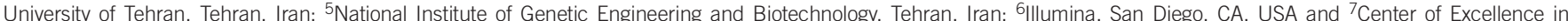
Phylogeny of Living Organisms in Iran, School of Biology, College of Science, University of Tehran, Tehran, Iran 
testing, mild elevation of methylmalonic acid (MMA) in the urine and plasma has often been reported, and it was suggested that MMA is the hallmark of encephalomyopathy due to mutations in SUCLA2. ${ }^{8}$ The elevation is a consequence of succinyl-CoA buildup, which occurs because SUCLA2 mutations prevent its conversion to succinate. The buildup in turn shifts the reversible reaction of conversion of succinyl$\mathrm{CoA}$ to methylmalonic-CoA in the direction of methylmalonic-CoA accumulation.

Mutations in SUCLG1 and SUCLA2 are rarely observed, consistent with the more general observation that genetic defects in the TCA cycle are uncommon and probably cause death before birth. ${ }^{18}$ SUCLA2 mutations have been reported in only 32 individuals, 23 of whom were from the genetically isolated Faroe Islands. The remaining nine belonged to four different families. ${ }^{8,19-21}$ Here, we describe identification of SUCLA2 as the causative gene of encephalomyopathy in two Iranian cousins and report a novel mutation in the gene.

\section{MATERIALS AND METHODS}

The research was performed in accordance with the Helsinki Declaration and with the approval of the ethics board of the University of Tehran. The responsible guardians of the participants consented to participate in the research.

\section{Subjects}

Patient 1 (DS-101-5). The patient is the second child of healthy consanguineous parents from Yazd in central Iran (Figure 1). Her elder sister is apparently well. The patient was born by cesarian section with a birth weight of $2.9 \mathrm{~kg}$. Delayed motor milestone, failure to thrive and hearing loss were noticed by the parents by the second year of life. The child was not able to sit unaided or walk. She suffered from feeding problems, leading to failure to thrive. Her age at the time of study was 4 years. The child manifested hypotonia, severe generalized dystonia and encephalopathy (Supplementary Figure 1 and Supplementary videos). No external ophthalmoplegia and/or ptosis, and divergent strabismus were observed in the affected child. Additionally, no sign of epilepsy, cardiomyopathy and renal tubular dysfunction were seen in the child. T2-weighted and axial flair magnetic resonance images (MRI) of the patient's brain showed hyperintensity of caudate nuclei and putamen (Figures $2 \mathrm{a}$ and $\mathrm{b})$. Laboratory investigations showed increased blood lactate (23.8 $\mathrm{mg} \mathrm{dl}^{-1}$; normal 4.5-20). Blood ammonia and pyruvate were in the normal ranges. Amino-acid analysis in blood sample showed no elevation of

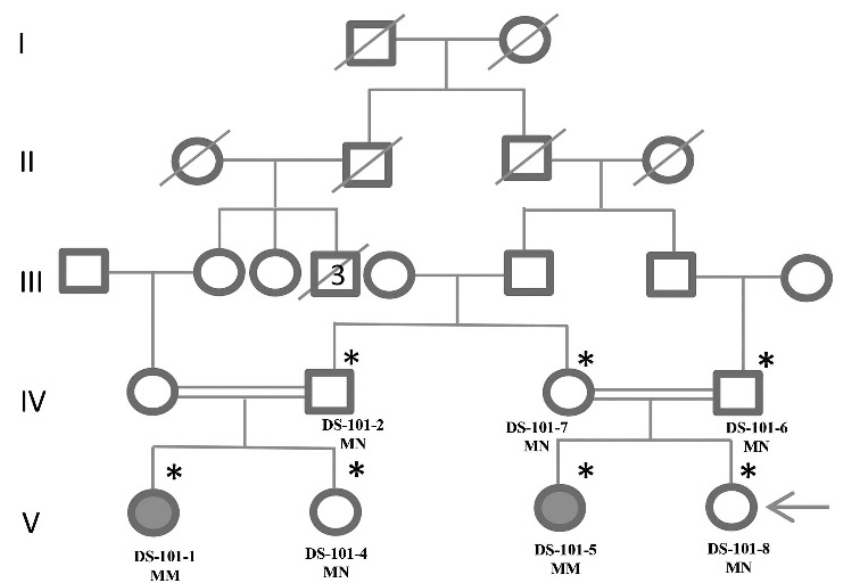

Figure 1 Pedigree with SUCLA2 mutation. Arrow: proband; filled circles: affected individuals; *: individuals included in the linkage analysis. Genotypes of individuals ascertained by direct sequencing are presented: N, normal SUCLA2 allele; M, mutated SUCLA2 allele. any amino acids. The organic acids in urine showed elevation of 4-hydroxyphenyllactic acid $(21 \mathrm{mmol}$ per mol creatinine; normal $<3.1)$. The acylcarnitine profile in blood showed increased level of succinylcarnitine (C4CD) $\left(2.00 \mu \mathrm{moll}^{-1}\right.$; normal $\left.0.08-1.5\right)$. Qualitative and quantitative measurements did not show increased MMA levels $(2 \mu \mathrm{mol}$ per mmol creatinine; normal $<5)$. The ceruloplasmin level was normal.

Patient 2 (DS-101-1). Patient 2 is a cousin of patient 1, and the four parents of the two affected children are all consanguineous with one another (Figure 1). The parents and the single sibling of patient 2 are healthy. Patient 2 was born by cesarian section with a birth weight of $2.7 \mathrm{~kg}$. As reported by the parents, the first symptom was hearing loss at age of 2 years. She was otherwise normal for $\sim 1$ year, when gait problems and abnormal postures (dystonia) appeared at the age of 3 years. The dystonia progressed slowly and she was later unable to walk unaided. She also had severe dysarthria and dysphagia due to severe bulbar dystonia. Her age at the time of study was 10 years. Brain T2weighted and axial flair MRI showed hyperintensity of both the putamen and caudate nuclei (Figures $2 \mathrm{c}$ and d). Laboratory investigations were not performed on this patient.

Controls. Two hundred ethnically matched healthy Iranian adults were recruited to serve as controls.

\section{Genome-wide genotyping and homozygosity mapping}

DNA was isolated according to standard phenol-chloroform methods. Genome-wide single-nucleotide polymorphism (SNP) genotyping was carried out using HumanCytoSNP-12v1-0_D BeadChips and the iScan reader (Illumina; www.illumina.com). Seven individuals were genotyped, including the two affected cousins, three unaffected parents, and one unaffected sibling of each patient (Figure 1). SNPs that had not been genotyped in one or more individual and SNPs that exhibited Mendelian error were removed from the analysis with appropriate options in the GenomeStudio V2010.3 program (Illumina). Homozygous regions common to the two affected individuals with a minimum physical length of $1 \mathrm{Mb}$ and absent in non-affected individuals were sought using the Homozygosity Detector tool within the GenomeStudio program. The SNP chip data output was subsequently exported to Microsoft Excel software and homozygous regions were confirmed within the EXCLUDEAR spreadsheet. Genomic positions are reported with reference to Human Genome Build 37.3.

\section{Mutation screening}

The thirteen exons and flanking intronic sequences of SUCLA2 in both affected children were amplified by polymerase chain reaction (PCR) and subsequently sequenced using the dye termination chemistry (Big Dye kit and the Prism 3700 sequencer; Applied Biosystems, Foster City, CA, USA). Sequences were analyzed with the Sequencher 4.8 software (Gene Codes, Ann Arbor, MI, USA). Sequence variations were assessed by comparison with reference sequences available at the National Center for Biotechnology Information: NT_024524.14, NM_003850.2, and NP_003841.1. Effects of variant sequences on splicing were predicted using NNsplice 0.9 (http://www.fruitfly.org/seq_ tools/splice.html). Having identified the putative disease-associated sequence variation in SUCLA2, the mutation status was assessed in the unaffected parents and siblings of the patients by direct sequencing. Subsequently, the variation was screened in 200 ethnically matched control individuals using an allele-specific PCR protocol. Sequences of all primers used are available upon request.

\section{In silico structural modeling}

The 3D structure of wild type and mutated forms of ADP-forming succinateCoA ligase were predicted through homology modeling and molecular dynamics simulation. The crystal structure of this protein is not presently available. Its amino-acid sequence (NP_003841.1) was used in a BLAST search of the Protein Data Bank (PDB; http://www.expasy.org/) to identify the protein with maximum homology among proteins with known 3D structures. The pig succinate-CoA ligase in complex with GTP (PDB code 2FPG) was identified (71\% sequence similarity), and its structure served as template for ADP- 
forming succinate-CoA ligase model building using the Modeller9v7 program $^{22}$ (http://salilab.org/modeller/). Out of 1000 models generated by Modeller for the encoded protein, the one with the best $G$-score was selected for energy minimization by molecular dynamics simulation using the GROMACS (gromacs-4.5.5 software; www.gromacs.org). ${ }^{23}$ For this purpose, the molecule was solvated with water molecules within a cubic box. The root mean square deviation (RMSD) for backbone atoms between the derived model and the template was estimated by GROMACS. Figure presentation was performed with VMD 1.9.1. ${ }^{24}$

\section{RESULTS}

The inheritance pattern of the disorder appeared autosomal recessive, as affected offsprings were born to consanguineous unaffected parents. The presentations in the affected children suggested a metabolic disorder possibly associated with mitochondrial dysfunction. ${ }^{25}$

The GenomeStudio Homozygosity Detector tool of the Genome Studio program identified only one region on chromosome 13 that was homozygous in both affected individuals and not homozygous in the unaffected parents and siblings (Supplementary Figure 2).
The common homozygous region expanded $15.5 \mathrm{Mb}$ and was bound by proximal and distal markers, respectively, rs9576228 (37819029 bp) and rs4885981 (53364443 bp). The locus includes 190 annotated protein genes, and SUCLA2 is one of the genes within the locus. Based on earlier phenotypic descriptions of patients harboring mutations in SUCLA2, this was considered a strong candidate disease-causing gene in our pedigree. ${ }^{8,19,20}$ Screening of this gene in the two patients by sequencing identified five homozygous sequence variations. Four of these were previously reported polymorphisms that were positioned outside the amino-acid coding sequences and did not affect splicing. The remaining variation, c.751G $>$ A, is novel and causes p.Asp251Asn in the encoded protein. Aspartic acid at position 251 is highly conserved during evolution, and remains unchanged in orthologous proteins of mammals, birds, fish and invertebrates, and in the paralogous human protein encoded by SUCLG2 (Supplementary Table 1). The PolyPhen (http://genetics.bwh.harvard.edu/pph2/), SIFT (http://sift.jcvi.org/), CONDEL (http://bg.upf.edu/condel/help/) and Mutpred (http://mutpred.mutdb.org/) bioinformatics tools predicted that p.Asp251Asn would damage protein function. Genetic analysis of parents and unaffected
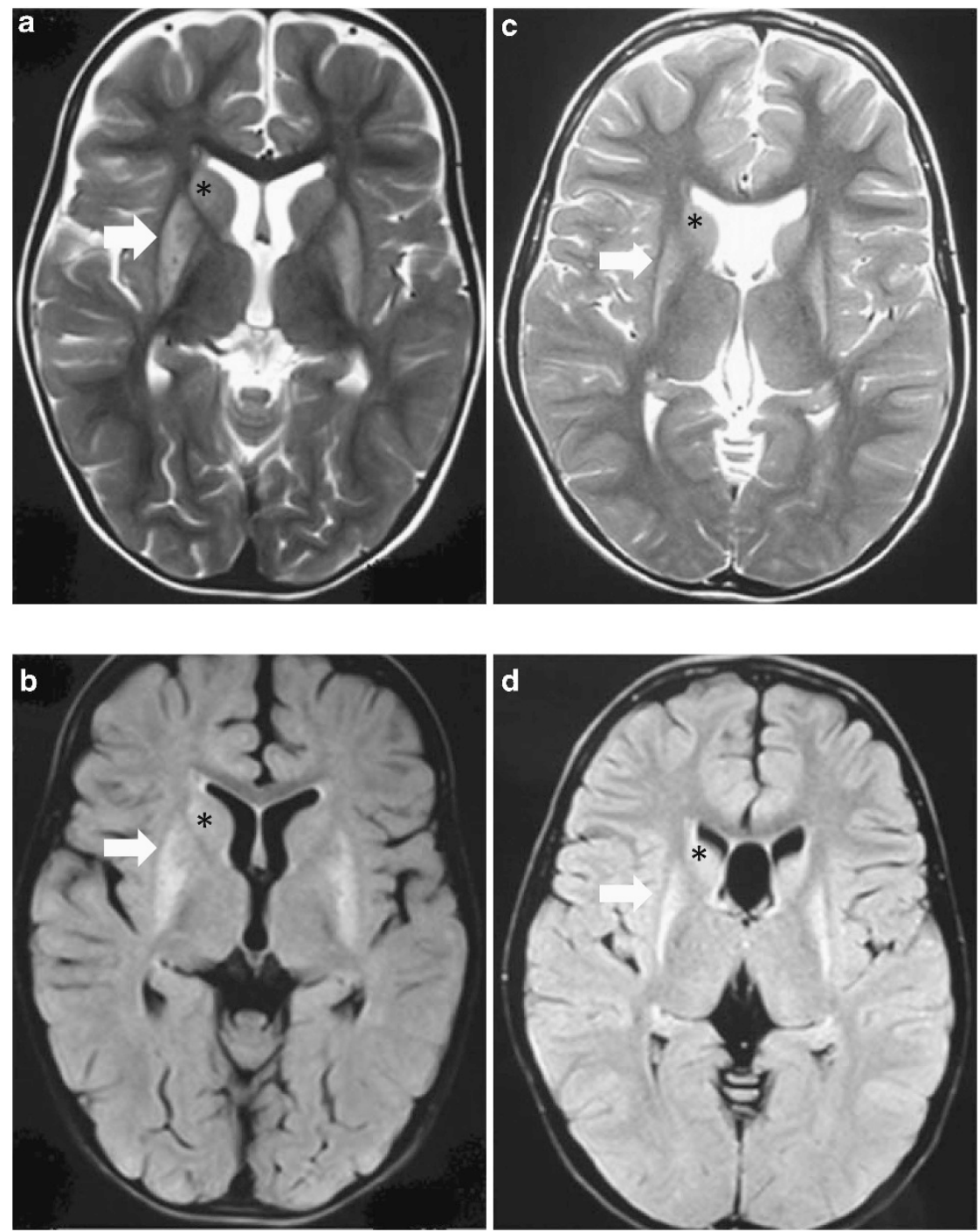

Figure 2 Brain magnetic resonance images. T2-weighted (a) and axial flair (b) images of the brain of the proband at the age of 4 years, and T2-weighted (c) and axial flair (d) images of the brain of the affected cousin at the age of 10 years. Hyperintensity in caudate nuclei (stars) and putamens (arrows) in the brain of both patients is evident. 
siblings of patients with these mutations indicated segregation with disease status (Supplementary Figure 3). Finally, the causative nucleotide variation was not observed in 200 ethnically matched individuals. It was concluded that c.751G $>\mathrm{A}$ in SUCLA2 that affects p.Asp251Asn was the disease-causing mutation in our pedigree.

The clinical features of the patients studied here closely resembled those of previously reported patients with mutations in SUCLA2. ${ }^{8,19-21}$ In addition to these features, the patients studied earlier exhibited mtDNA depletion in muscle tissue, specific anomalies in magnetic resonance images (MRI) of the brain, and a biochemical profile that included lactate acidosis and mildly elevated levels of MMA in the urine. Muscle tissue from our proband was not available for mtDNA evaluation. MRI images of both the proband and her affected cousin showed characteristic hyperintensity of caudate nuclei and putamen (Figure 2). Lactate acidosis in the plasma and elevated 4-hydroxyphenyllactic acid in the urine of the proband were detected. However, increased MMA levels were not observed, and instead an increase in the level of blood C4DC was reported.
In silico structural analysis was performed to obtain insight into the structural effects of the four known disease-associated amino-acid variations in the $\beta$-subunit of succinate-CoA ligase, including p.Asp251Asn observed in this study. Based on conserved domain search in NCBI (http://www.ncbi.nlm.nih.gov/Structure/cdd/ cdd.shtml), p.Ala103, p.Gly118 and p.Asp251 among the affected amino acids are positioned within the ATP-grasp domain, and p.Arg284 is situated in the linking region between the ATP-grasp domain and the ligase-CoA domain (Figure 3a). Dynamic modeling of the structure produced by Modeller resulted in a structure whose RMSD reached a plateau of $0.3 \mathrm{~nm}$ after $5 \mathrm{~ns}$, indicating the reliability of the predicted structure. Superimposition of the modeled structure of the encoded $\beta$-subunit on the dimeric structure of template pig succinate-CoA ligase is shown in Figure $3 \mathrm{~b}$. The positions of amino acids affected by four known missense mutations are also shown. P.Asp251 is positioned within the region of intersection between $\alpha$ - and $\beta$-subunits of the template protein, suggesting that it may affect interactions between the two subunits. Figures $3 \mathrm{c}-\mathrm{f}$ show

a

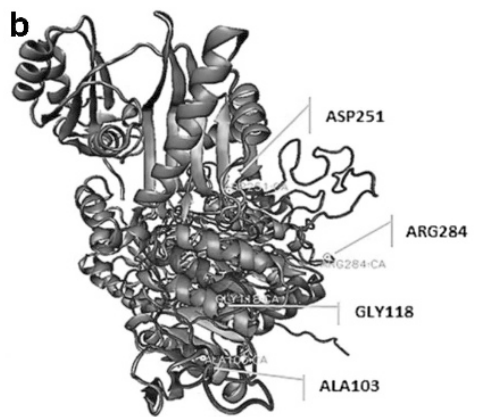

C

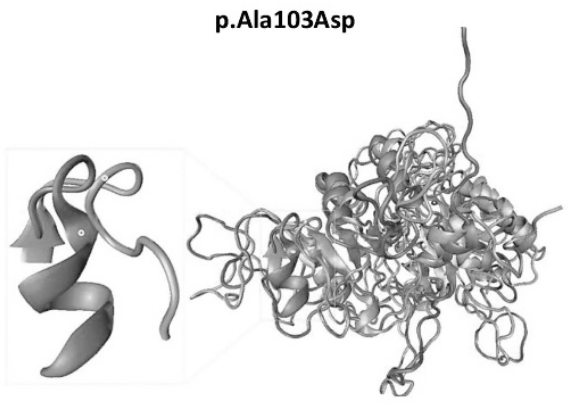

e

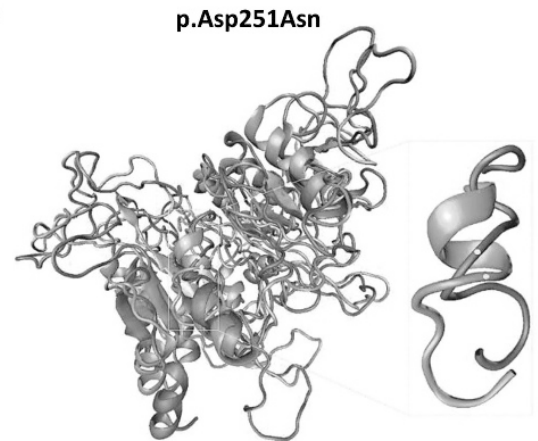

d p.Gly118Arg

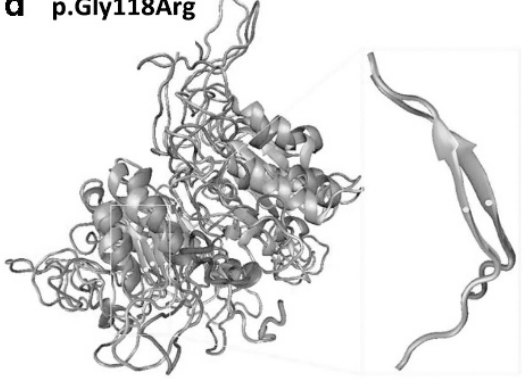

f p.Arg284Cys

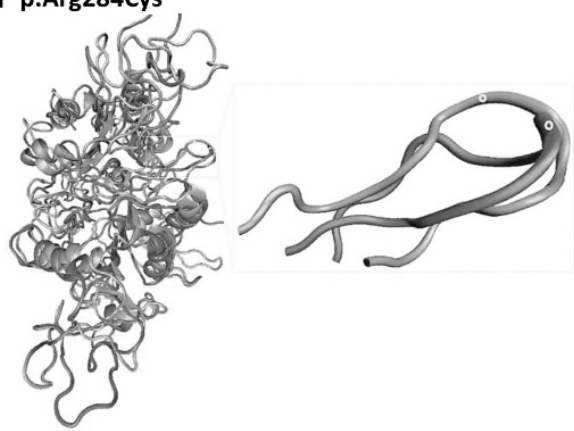

Figure 3 Analysis of the effects of SUCLA2 missense mutations on the structure of encoded protein using Modeller and GROMACS. (a) Predicted positions of amino acids affected by four known missense mutations based on NCBI conserved domain search. (b) Modeled structure of the encoded $\beta$-subunit (red) superimposed on the dimeric structure of the template structure of template pig succinate-CoA ligase (blue). Positions of amino acids affected by four known missense mutations are shown. (c-f) Modeled structure of, respectively, p.Ala103Asp, p.Gly118Arg, p.Asp251Asn and p.Arg284Cys (blue) mutated proteins superimposed on themodeled structure of wild type (red). Enlargements of regions containing the affected amino acids are also shown. Predicted structural changes caused by the mutations are described in text. RMSD values for modeled mutated and wild-type proteins ranged from 0.61 to $0.68 \mathrm{~nm}$. A full color version of this figure is available at the Journal of Human Genetics journal online. 
superimpositions of modeled structures of mutated proteins on the modeled structure of wild type. P.Ala103Asp results in transformation of an $\alpha$-helix to a loop (Figure 3c); p. Asp251Asn causes a loop that includes p.251 to form an $\alpha$-helix (Figure 3e); and p.Arg284Cys causes a change in the direction of the loop connecting the ATP-grasp domain and the ligase-CoA domain (Figure 3f). It is expected that these structural changes may affect protein function. P.Gly118Arg did not cause an obvious structural change (Figure $3 \mathrm{~d}$ ).

\section{DISCUSSION}

We report finding the novel disease-causing mutation c.751G $>$ A in SUCLA2 that affects p.Asp251Asn in the encoded protein in two cousins affected with encephalomyopathy. The causative locus was identified by genome-wide linkage analysis, and the causative mutation was discovered by candidate gene sequencing. The variation is considered to be cause of the disease based on very high evolutionary conservation of the affected amino acid, segregation with disease status in the pedigree, bioinformatics-based predictions of the effect of the altered amino acid on function and structure, and absence of the mutation in 200 control individuals. Structural modeling results, based on a structure of a template protein with $71 \%$ sequence similarity, suggested that the mutated amino acid induces a structural change.

Previously, only five mutations in SUCLA2 were documented, two that affect splicing and three that cause amino-acid alterations. ${ }^{8,19-21}$ One patient harbored compound heterozygous mutations, and the rest carried homozygous mutations. The phenotypic presentation in the Iranian patients was generally similar to that of previously described patients with mutations in SUCLA2.

Although elevated MMA has been considered the biochemical hallmark of patients with mutations in SUCLA2, we did not observe elevated MMA in the urine of our proband. We observed mild elevation of C4DC. These findings are not unique to our proband. Absence of elevated MMA and presence of elevated C4DC have occasionally been reported in other patients harboring SUCLA2 mutations. $^{20,21}$ This elevation is probably also a consequence of succinyl-CoA buildup, which can result in shift of the reversible conversion of succinyl-CoA to C4DC in the direction of C4DC accumulation. ${ }^{20}$ Elevated levels of C4-dicarboxylic carnitine, which may be a mixture of methylmalonylcarnitine and succinylcarnitine, have occasionally been reported in patients with SUCLA2 and SUCLG1 mutations..$^{20,26}$ We suspect that mild elevation in MMA or succinylcarnitine or both may occur in patients with SUCLA2 mutations, but that the elevations may not be evident at all time points and repeated measurements may be necessary to observe the elevations. In any case, elevated MMA is not always observed in encephalopathic patients with mtDNA depletion and mutations in SUCLA2, and this should be taken into account in making genetic diagnoses.

\section{ACKNOWLEDGEMENTS}

We thank the patients and their family members for consenting to participate in this study. We acknowledge the Tehran University of Medical Sciences and the Research Council of the University of Tehran for funding this research.
1 Chinnery, P. F. \& Turnbull, D. M. Epidemiology and treatment of mitochondrial disorders. Am. J. Med. Genet. 106, 94-101 (2001).

2 Zeviani, M. \& Di Donato, S. Mitochondrial disorders. Brain 127, 2153-2172 (2004).

3 Coenen, M. J., Antonicka, H., Ugalde, C., Sasarman, F., Rossi, R., Heister, J. G. et al. Mutant mitochondrial elongation factor G1 and combined oxidative phosphorylation deficiency. N. Engl. J. Med. 351, 2080-2086 (2004).

4 Valente, L., Tiranti, V., Marsano, R. M., Malfatti, E., Fernandez-Vizarra, E., Donnini, C. et al. Infantile encephalopathy and defective mitochondrial DNA translation in patients with mutations of mitochondrial elongation factors EFG1 and EFTu. Am. J. Hum. Genet. 80, 44-58 (2007).

5 Davis, R. L. \& Sue, C. M. The genetics of mitochondrial disease. Semin Neurol. $\mathbf{3 1}$ 519-530 (2011).

6 Scaglia, F. Nuclear gene defects in mitochondrial disorders. Methods Mol. Biol. 837 17-34 (2012).

7 Copeland, W. C. Defects in mitochondrial DNA replication and human disease. Crit Rev. Biochem. Mol. Biol. 47, 64-74 (2012).

8 Elpeleg, O., Miller, C., Hershkovitz, E., Bitner-Glindzicz, M., Bondi-Rubinstein, G., Rahman, S. et al. Deficiency of the ADP-forming succinyl-CoA synthase activity is associated with encephalomyopathy and mitochondrial DNA depletion. Am. J. Hum. Genet. 76, 1081-1086 (2005)

9 Spinazzola, A. \& Zeviani, M. Disorders from perturbations of nuclear-mitochondrial intergenomic cross-talk. J. Intern. Med. 265, 174-192 (2009).

10 Johnson, J. D., Mehus, J. G., Tews, K., Milavetz, B. I. \& Lambeth, D. O. Genetic evidence for the expression of ATP- and GTP-specific succinyl-CoA synthetases in multicellular eucaryotes. J. Biol. Chem. 273, 27580-27586 (1998).

11 Jeffery, C. J. Moonlighting proteins. Trends Biochem. Sci. 24, 8-11 (1999).

12 Tomlinson, I. P., Alam, N. A., Rowan, A. J., Barclay, E., Jaeger, E. E., Kelsell, D. et al. Germline mutations in $\mathrm{FH}$ predispose to dominantly inherited uterine fibroids, skin leiomyomata and papillary renal cell cancer. Nat. Genet. 30, 406-410 (2002).

13 Miller, C., Wang, L., Ostergaard, E., Dan, P. \& Saada, A. The interplay between SUCLA2, SUCLG2, and mitochondrial DNA depletion. Biochim. Biophys. Acta 1812 625-629 (2011)

14 Lambeth, D. O., Tews, K. N., Adkins, S., Frohlich, D. \& Milavetz, B. I. Expression of two succinyl-CoA synthetases with different nucleotide specificities in mammalian tissues. J Biol Chem. 279, 36621-36624 (2004).

15 Ostergaard, E., Schwartz, M., Batbayli, M., Christensen, E., Hjalmarson, O., Kollberg G. et al. A novel missense mutation in SUCLG1 associated with mitochondrial DNA depletion, encephalomyopathic form, with methylmalonic aciduria. Eur. J. Pediatr. 169, 201-205 (2010).

16 Kavanaugh-Black, A., Connolly, D. M., Chugani, S. A. \& Chakrabarty, A. M. Characterization of nucleoside-diphosphate kinase from Pseudomonas aeruginosa: complex formation with succinyl-CoA synthetase. Proc. Natl. Acad. Sci. USA 91, 5883-5887 (1994).

17 Kowluru, A., Tannous, M. \& Chen, $\mathrm{H}$. Q Localization and characterization of the mitochondrial isoform of the nucleoside diphosphate kinase in the pancreatic beta cell: evidence for its complexation with mitochondrial succinyl-COA synthetase. Arch. Biochem. Biophys. 398, 160-169 (2002).

18 Rustin, P., Bourgeron, T., Parfait, B., Chretien, D., Munnich, A. \& Rotig, A. Inborn errors of the Krebs cycle: a group of unusual mitochondrial diseases in human. Biochim. Biophys. Acta 1361, 185-197 (1997).

19 Ostergaard, E., Hansen, F. J., Sorensen, N., Duno, M., Vissing, J., Larsen, P. L. et al. Mitochondrial encephalomyopathy with elevated methylmalonic acid is caused by SUCLA2 mutations. Brain 130, 853-861 (2007).

20 Carrozzo, R., Dionisi-Vici, C., Steuerwald, U., Lucioli, S., Deodato, F., Di Giandomenico, S. et al. SUCLA2 mutations are associated with mild methylmalonic aciduria, Leigh-like encephalomyopathy, dystonia and deafness. Brain 130, 862-874 (2007).

21 Lamperti, C., Fang, M., Invernizzi, F., Liu, X., Wang, H., Zhang, Q. et al. A nove homozygous mutation in SUCLA2 gene identified by exome sequencing. Mol. Genet. Metab. 107, 403-408 (2012)

22 Sali, A. \& Blundell, T. L. Comparative protein modelling by satisfaction of spatial restraints. J. Mol. Biol. 234, 779-815 (1993).

23 Van Der Spoel, D., Lindahl, E., Hess, B., Groenhof, G., Mark, A. E. \& Berendsen, H. J. GROMACS: fast, flexible, and free. J. Comput. Chem. 26, 1701-1718 (2005).

24 Humphrey, W., Dalke, A. \& Schulten, K. VMD: visual molecular dynamics. J. Mol. Graph. 14, 27-38 (1996).

25 Spinazzola, A., Invernizzi, F., Carrara, F., Lamantea, E., Donati, A., Dirocco, M. et al. Clinical and molecular features of mitochondrial DNA depletion syndromes. J. Inherit. Metab. Dis. 32, 143-158 (2009).

26 Rouzier, C., Le Guedard-Mereuze, S., Fragaki, K., Serre, V., Miro, J., Tuffery-Giraud, S. et al. The severity of phenotype linked to SUCLG1 mutations could be correlated with residual amount of SUCLG1 protein. J. Med. Genet. 47, 670-676 (2010).

Supplementary Information accompanies the paper on Journal of Human Genetics website (http://www.nature.com/jhg) 